\title{
Rare Branching Pattern of the Subscapular Artery
}

\author{
Alexey Vladimirovich Tverskoi ${ }^{1}$ Vitaly Nikolaevich Morozov ${ }^{1}$ Svetlana Aleksandrovna Petrichko ${ }^{1}$ \\ Vitaly Vladimirovich Pushkarskiy ${ }^{2}$ Aleksandr Sergeevich Parichuk ${ }^{2}$
}

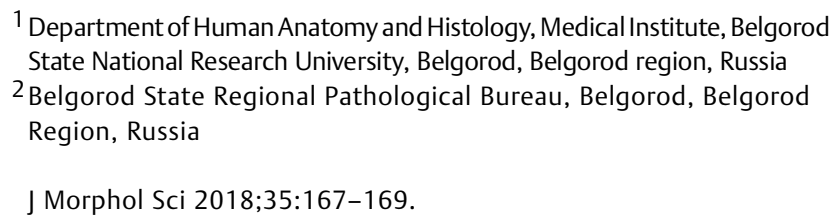

\author{
Address for correspondence Vitaly Nikolaevich Morozov, \\ Department of Human Anatomy and Histology, Medical Institute, \\ Belgorod State National Research University, Pobedy Street, 85, \\ Belgorod, Belgorod Region, 308015, Russia \\ (e-mail: vitaliyy-morozov@rambler.ru).
}

\begin{abstract}
Variations of the axillary artery and its branches are quite common. Some variations are clinically significant and having knowledge of them can be useful for the prevention of diagnostic errors during surgical interventions in the axillary fossa. Classically, the third part of the axillary artery presents three branches-the subscapular, the anterior, and the posterior circumflex humeral arteries. The subscapular artery is divided into the circumflex scapular and the thoracodorsal arteries. Our work presents a previously undescribed branching pattern of the right subscapular artery. It branched into the thoracodorsal, the circumflex scapular, the profunda brachii, and the anterior and posterior circumflex humeral arteries. The profunda brachii artery was $0.4 \mathrm{~cm}$ in

Keywords

- artery

- axillary

- subscapular

- variation diameter and ran inferiorly to the humeromuscular canal. No branches of the profunda brachii artery were found in the superior part of the arm before entering the humeromuscular canal. No variations in the other parts of the right axillary artery and of the left axillary artery were discovered. The described branching pattern of the subscapular artery can be important and essential for surgeons and radiologists.
\end{abstract}

\section{Introduction}

The embryonic development of blood vessels is a complex process, which results in variable levels of origin, course and branching. ${ }^{1}$ It is of theoretical and clinical interest for anatomists, cardiac and vascular surgeons, and radiologists.

The axillary artery is the continuation of the subclavian artery from the outer border of the first rib. The axillary artery is classically divided into three parts at the level of the pectoralis minor muscle. The first part (proximal) begins at the lateral border of the pectoralis minor muscle. The second part (posterior) of the axillary artery lies deep in the pectoralis minor muscle. The third part (distal) lies between the lateral border of the pectoralis minor muscle and the inferior border of the teres major muscle. The typical axillary artery is supposed to give origin to six branches in three parts. The first part of the artery branches into the superior thoracic and the thoracoacromial arteries. The second part branches into the lateral thoracic artery. The third part branches into received

May 30, 2018

accepted

September 6, 2018

published online

October 31, 2018 the subscapular, the anterior circumflex humeral, and the posterior circumflex humeral arteries. ${ }^{2}$

Variations in the beginning, in the course and in the branching of the subscapular artery are widely represented in the works of other anatomists. ${ }^{3-5}$ The present work shows a rare branching pattern of the subscapular artery, which was observed unilaterally in a female cadaver.

\section{Case Report}

During a routine dissection in a 72-year-old female cadaver at the department of human anatomy and histology of the Belgorod State National Research University, we observed unilateral variations in the branching pattern of the third part of the right axillary artery (-Fig. 1). No variations in the artery were discovered. The subscapular artery was $0.7 \mathrm{~cm}$ in length. It branched into the thoracodorsal, the circumflex scapular, the profunda brachii, the anterior and the posterior other parts of the right axillary artery and of the left axillary
Copyright (e 2018 by Thieme Revinter Publicações Ltda, Rio de Janeiro, Brazil

\section{License terms}

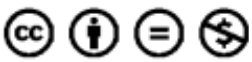




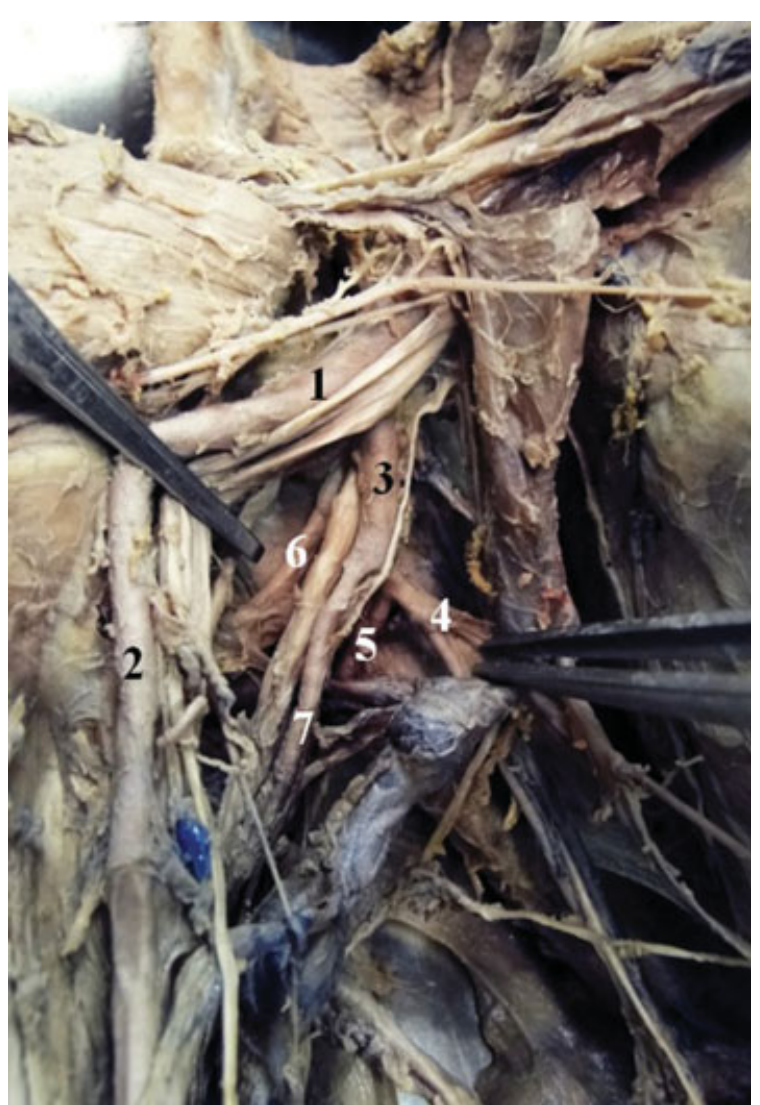

Fig. 1 Right axillary cavity showing the origin of the subscapular artery and its branches. 1-Axillary artery; 2-Brachial artery; 3-Subscapular artery; 4-Thoracodorsal artery; 5-Circumflex scapular artery; 6-Posterior circumflex humeral artery; 7-Profunda brachii artery.

circumflex humeral arteries. The profunda brachii artery was $0.4 \mathrm{~cm}$ in diameter and ran inferiorly to the humeromuscular canal. No branches of the profunda brachii artery were found in the superior part of the arm before entering the humeromuscular canal.

\section{Discussion}

Variations of the arteries of the upper limb, particularly in the axillary and in the subscapular arteries, are widely reported by other anatomists.

According to report of Saralaya V. (2008), the first part of the axillary artery divided into an unusual collateral branch, called the common subscapular trunk. It further branched into the circumflex scapular, the posterior circumflex humeral, the thoracodorsal, the thoracoacromial, and the lateral thoracic arteries. ${ }^{4}$

During a routine dissection in a 30-year-old male cadaver, a bilateral variation of the subscapular artery was observed. The right subscapular artery arose from the first part of the axillary artery and branched into the lateral thoracic, the thoracodorsal, and the posterior circumflex humeral arteries. The circumflex scapular artery arose from the posterior circumflex humeral artery, and the anterior circumflex humeral artery arose from the third part of the axillary artery. The left subscapular artery branched into the lateral thoracic, the thoracodorsal, and the circumflex scapular arteries. The anterior and posterior circumflex humeral arteries arose from the third part of the axillary artery. ${ }^{6}$

During the dissection of a male cadaver, a common subscapular trunk was observed, which branched into the circumflex scapular, the thoracodorsal, the lateral thoracic, and the posterior circumflex humeral arteries. ${ }^{5}$

According to report of Bhat KM. et al. (2008), the second part of the right axillary artery branched into the common trunk, which further branched into two muscular branches, supplying the pectoralis major and deltoid muscles, as well as the thoracoacromial, the lateral thoracic, and the subscapular arteries. The latter branched into the posterior circumflex humeral artery and into the common trunk, which branched into the thoracodorsal and the circumflex scapular arteries. ${ }^{3}$

The branching pattern of the subscapular artery that was most identical to that represented in our work was described in a case in which the right axillary artery divided into a large collateral branch (called the common subscapular trunk), which branched into the subscapular, the anterior and posterior circumflex humeral, the profunda brachii, and the superior ulnar collateral arteries in a male cadaver. ${ }^{7}$

A similar branching pattern of the left subscapular artery in a male cadaver was reported. ${ }^{8}$

In our work, the branching pattern of the subscapular artery somewhat differs from the last two cases described above. First, the subscapular artery arose directly from the third part of the axillary artery, but not from the common subscapular trunk. Second, it did not branch into the superior ulnar collateral artery; and third, the subscapular artery branched into the thoracodorsal artery.

\section{Conclusion}

In our work, we have described a rare case of the branching pattern of the subscapular artery, which differs from previous reports. The knowledge of a rare variation in the axillary artery and its branches is required for the identification of stenotic lesions, for the determination of the possibility of collateral circulation, as well as for endovascular manipulation, including contrast enhanced computed tomography angiography, ultrasound and magnetic resonance imaging. This can be useful for the prevention of diagnostic errors during surgical interventions in the axillary fossa.

\section{References}

1 Adair TH, Montani JP. Angiogenesis. Morgan \& Claypool Life Sciences; 2010; 84

2 Drake RL, Vogl AW, Mitchell AW. Gray's Anatomy for Students. Philadelphia: Elsevier; 2015:1161

3 Bhat KM, Gowda S, Potu BK, Rao MS. A unique branching pattern of the axillary artery in a South Indian male cadaver. Bratisl Lek Listy (Tlacene Vyd) 2008;109(12):587-589

4 Saralaya V, Joy T, Madhyastha S, Vadgaonkar R, Saralaya S. Abnormal branching of the axillary artery: subscapular common trunk. A case report. Int J Morphol 2008;26(04):963-966

5 Dimovelis I, Michalinos A, Spartalis E, Athanasiadis G, Skandalakis $\mathrm{P}$, Troupis T. Tetrafurcation of the subscapular artery. Anatomical 
and clinical implications. Folia Morphol (Warsz)2016https:// journals.viamedica.pl/folia_morphologica/article/view/48381

6 Pushpalatha M, Chitra N. Variations in subscapular artery: a study. J Evid Based Med Healthcare 2015;2(04):308-312
7 Venieratos D, Lolis ED. [Abnormal ramification of the axillary artery: sub-scapular common trunk]. Morphologie 2001;85(270):23-24

8 Jain A, Kumar MS. An unusual variation of axillary artery: a case report. J Clin Diagn Res 2015;9(01):AD05-AD07 